\title{
Correction to: Long non-coding RNA MALAT1 regulates trophoblast functions through VEGF/VEGFR1 signaling pathway
}

\author{
Chun Feng ${ }^{1} \cdot$ Lin Cheng ${ }^{2} \cdot$ Jing Jin $^{3} \cdot$ Xiaoxia Liu $^{3} \cdot$ Fang Wang ${ }^{2}$ (])
}

Published online: 9 August 2021

○) Springer-Verlag GmbH Germany, part of Springer Nature 2021

\section{Correction to: Archives of Gynecology and Obstetrics https://doi.org/10.1007/s00404-021-05987-y}

In the original article published, the affiliation of the first author has been changed. The correct affiliation is "Department of Gynecology, Maternal and Child Health Hospital of Hubei Province, Tongji Medical College, Huazhong University of Science and Technology".

Publisher's Note Springer Nature remains neutral with regard to jurisdictional claims in published maps and institutional affiliations.

The original article can be found online at https://doi.org/10.1007/ s00404-021-05987-y.

Fang Wang

wonfun@whu.edu.cn

1 Department of Gynecology, Maternal and Child Health Hospital of Hubei Province, Tongji Medical College, Huazhong University of Science and Technology, Wuhan, China

2 Department of Obstetrics and Gynecology, Zhongnan Hospital, Wuhan University, Wuhan 430071, Hubei, China

3 Department of Obstetrics and Gynecology, Union Hospital, Tongji Medical College, Huazhong University of Science and Technology, Wuhan 430022, Hubei, China 